\title{
Labyrinthe
}

22 | 2005 (3)

La Biopolitique (d')après Michel Foucault

\section{La découverte de la liberté}

\section{Diogo Sardinha}

\section{OpenEdition}

\section{Journals}

Édition électronique

URL : http://journals.openedition.org/labyrinthe/1037

DOI : $10.4000 /$ labyrinthe. 1037

ISSN : 1950-6031

Éditeur

Hermann

Édition imprimée

Date de publication : 1 novembre 2005

Pagination : 89-99

Référence électronique

Diogo Sardinha, «La découverte de la liberté », Labyrinthe [En ligne], 22 | 2005 (3), mis en ligne le 22 juillet 2008, consulté le 02 mai 2019. URL : http://journals.openedition.org/labyrinthe/1037 ; DOI : 10.4000/labyrinthe. 1037

Propriété intellectuelle 


\title{
LA DÉCOUVERTE DE LA LIBERTÉ
}

\author{
Diogo SARDINHA \\ diogo_pt@hotmail.com
}

Si la liberté est longtemps restée pour Foucault un sujet mineur, c'est qu'il ne l'avait jamais vraiment découverte. En 1966, Les Mots et les choses font état de «notre liberté si soumise ${ }^{1} »$. Dix ans plus tard, La volonté de savoir nous apprend que le dispositif de sexualité «nous fait croire qu'il y va de notre "libération" ", alors qu'il y va de notre asservissement (VS, «Tel», p. 211). Cependant, un texte de 1982 fait apparaître la liberté non seulement comme une condition de possibilité de l'existence du pouvoir, mais encore comme un élément essentiel à l'exercice de ce dernier: pas de pouvoir sans liberté, écrit Foucault deux ans avant sa mort ${ }^{2}$. D'où vient ce changement? Où Foucault a-t-il trouvé la liberté, pour qu'elle devienne tellement décisive que sans elle on ne comprendra plus ses dernières réflexions sur le pouvoir? Les deux cours au Collège de France renferment la réponse à ces questions.

\section{Trois fausses définitions et leurs conséquences}

Sécurité, territoire, population s'ouvre par un retour au passé: «j'ai dit quelque part qu'on ne pouvait pas comprendre la mise en place des idéologies et d'une politique libérales au XVIII' siècle sans bien garder à l'esprit que ce même XVIII ${ }^{\mathrm{e}}$ siècle, qui avait si fortement revendiqué les libertés les avait tout de même lestées d'une technique disciplinaire qui, prenant les enfants, les soldats, les ouvriers là où ils étaient, limitait considérablement la liberté et donnait en quelque sorte des garanties à l'exercice même de cette liberté. » (STP, p. 50.) Foucault fait allusion à son livre de 1975, Surveiller et Punir, où il avait écrit en quatrième de couverture que « le XVIII ${ }^{e}$ siècle a sans doute inventé les libertés; mais il leur a

\footnotetext{
1. Paris, Gallimard, p. 224.

2. Dans « Le sujet et le pouvoir», DE, t. IV, n 306, p. 238.
} 
donné un sous-sol profond et solide - la société disciplinaire dont nous relevons toujours». À peine trois ans plus tard, Foucault revient sur ses pas: « Eh bien, je crois que j'ai eu tort. Je n'ai jamais tout à fait tort, bien sûr, mais enfin, ce n'est pas exactement ça. Je crois que ce qui est en jeu, c'est tout autre chose. C'est qu'en fait cette liberté, à la fois idéologie et technique de gouvernement, cette liberté doit être comprise à l'intérieur des mutations et transformations des technologies de pouvoir. Et, d'une façon plus précise et particulière, la liberté n'est pas autre chose que le corrélatif de la mise en place des dispositifs de sécurité. Un dispositif de sécurité ne peut bien marcher, et en tout cas celui dont je vous ai parlé là, qu'à la condition, justement, que l'on donne quelque chose qui est la liberté, au sens moderne [que ce mot] prend au XVIII siècle: non plus les franchises qui sont attachées à une personne, mais la possibilité de mouvement, déplacement, processus de circulation et des gens et des choses. Et c'est cette liberté de circulation, au sens large du terme, c'est cette faculté de circulation qu'il faut entendre, je crois, par le mot de liberté, et la comprendre comme étant une des faces, un des aspects, une des dimensions de la mise en place des dispositifs de sécurité.» (STP, p. 50.)

Il y a ainsi deux voies pour penser les liens entre le pouvoir et la liberté. La première est celle des libertés lestées par, ou fondées sur, les disciplines, vieux schème foucaldien selon lequel les libertés sont la surface visible d'une réalité qui pénètre loin dans l'épaisseur de la société, jusqu'à détenir la vérité de ces libertés. Selon ce schème, les pouvoirs microphysiques instaurent au niveau des petites institutions (école, prison, hôpital, armée) la base - disciplinaire et normalisatrice des libertés que consacreront la loi générale et les institutions macrophysiques. Une seconde voie, Foucault l'annonce maintenant, est celle des libertés qui n'appartiennent plus simplement à l'immédiateté du monde politique, mais qui, au contraire, étant la condition de possibilité du fonctionnement des dispositifs qui permettent de gouverner, jouent un rôle à part entière dans ce monde. De ce point de vue, la découverte de la liberté, quoique réduite à une « simple » capacité de circulation des biens et des gens, représente un changement considérable dans la manière dont Foucault envisage le domaine du pouvoir. Première leçon à retenir, donc: le biopouvoir n'est pas l'espace dominé par la répression; il est celui de l'existence de la liberté.

On nous dira que les disciplines et les dispositifs de pouvoir-savoir ne se développaient pas davantage dans l'élément de la répression. Pour 


\section{La découverte de la liberté}

preuve, l'attaque contre l'hypothèse répressive dès les premières pages de La volonté de savoir. Ce serait méconnaître que ce qui s'oppose à la répression n'est aucunement la liberté, mais la production: de discours, de pratiques, d'habitudes, de savoirs, de pouvoirs. Le discours sur le sexe est certes intarissable, précisément là où on le croirait rare et austère, mais il n'y va pas, en lui, de notre libération. Dans Surveiller et Punir, le pendant des disciplines est le modèle carcéral. Aussi Foucault ne s'étonne-t-il plus que « la prison ressemble aux usines, aux écoles, aux casernes, aux hôpitaux, qui tous ressemblent aux prisons ${ }^{3}{ }$. Impossible donc de prétendre que la découverte des disciplines en soit aussi celle des libertés. Au contraire, là où la conscience moderne cachait les premières pour se présenter comme l'âge des secondes, Foucault parcourt le chemin en sens inverse et met au jour les disciplines. Le sens de cellesci est la prison, il n'est pas la liberté. $\rightarrow$ Voir l'article de L. Loiseau.

En contrepartie, le sens de la biopolitique est d'être le lieu de reconnaissance de la liberté, non plus comme une simple surface, mais comme un élément « à l'intérieur des mutations et transformations des technologies de pouvoir », un élément de ces technologies. C'est, pour Foucault, une découverte formidable. Toutefois, l'ardeur de cette trouvaille est vite tempérée par une première définition: «La liberté n'est pas autre chose que le corrélatif de la mise en place des dispositifs de sécurité. » Définition doublement négative, qui appelle une lecture positive: la liberté est ce corrélatif. Si bien que, au lieu de lui reconnaître un statut propre, Foucault la recouvre une seconde fois, non plus d'une couche de disciplines, mais d'un ensemble de dispositifs. À l'antéposition première des disciplines aux libertés, typique de Surveiller et Punir et de La volonté de savoir, se substitue désormais l'antéposition de la sécurité à ces mêmes libertés. La découverte de la liberté dans le cadre de la biopolitique ne semble que bien éphémère.

Et pourtant, sa répercussion se fera sentir tout au long des deux cours. Par elle se termine Sécurité, territoire, population (p. 359 sqq.); et avec elle débute Naissance de la biopolitique, qui dès sa première leçon fait le rapprochement entre la liberté et le libéralisme ( $N B$, p. 25). Deux leçons plus tard, l'enjeu du cours devient manifeste, quand la liberté reçoit deux nouvelles « définitions ».

3. SP, p. 229. 
Primo, « la liberté, ce n'est jamais rien d'autre - mais c'est déjà beaucoup - qu'un rapport actuel entre gouvernants et gouvernés, un rapport où la mesure du "trop peu" de liberté qui existe est donné par le "encore plus" de liberté qui est demandé » (NB, p. 64). Fausse définition, on le voit, dans la mesure où le mot défini est employé dans la définition ellemême. Foucault suppose donc que ses auditeurs savent déjà de quoi il parle, ce qui, étant donné la plurivocité du mot en question, n'est que trop problématique.

Secundo, et dans le cadre spécifique du régime libéral, « la liberté [...] n'est pas une donnée, la liberté n'est pas une région toute faite qu'on aurait à respecter, ou si elle l'est, ce n'est que partiellement, régionalement, dans tel ou tel cas, etc. La liberté, c'est quelque chose qui se fabrique à chaque instant» $(N B$, p. 66). D'où découle une «définition » du régime auquel convient cette liberté : «Le libéralisme, ce n'est pas ce qui accepte la liberté. Le libéralisme, c'est ce qui se propose de la fabriquer à chaque instant, de la susciter et de la produire avec bien entendu [tout l'ensemble] de contraintes, de problèmes de coût que pose cette fabrication. » On est là simultanément dans la continuité de Surveiller et Punir et de La volonté de savoir et dans la rupture avec eux $\rightarrow$ voir l'article de R. Nigro $\nleftarrow$. Dans la continuité, parce que le paradigme de la production demeure dominant et s'oppose à celui de la répression. Mais dans la discontinuité aussi, puisque ce qui est produit n'est plus un sujet assujetti, mais ce sont des libertés d'agir ou de se déplacer.

Il est essentiel de saisir les deux alternatives selon lesquelles raisonne Foucault. La première alternative pose la liberté soit comme une donnée, soit comme une production ${ }^{4}$. Or, face à ces deux options, il conviendrait de se demander si la liberté ne serait pas avant tout quelque chose qui se conquiert, d'ailleurs en accord avec la seconde « définition » qu'a introduite Foucault (d'après laquelle il y a toujours un «"encore plus" de liberté qui est demandé»), et qu'il semble avoir oubliée. La seconde alternative oppose l'universel d'une définition à l'absence pure et simple de définition. En effet, si Foucault ne veut pas « dire que la quantité de liberté [a] augmenté entre le début du XVIII ${ }^{\mathrm{e}}$ siècle et, disons, le XIX ${ }^{\mathrm{e}}$ » (NB, p. 64), c'est aussi « qu'il ne faut pas considérer que la liberté, ce

4. C'est ce qui frappe à la lecture de projet de Constitution européenne, là où « l'Union offre à ses citoyennes et citoyens un espace de liberté, de sécurité et de justice sans frontières intérieures, et un marché unique où la concurrence est libre et non faussée » (Art. I-3, 2, je souligne). $\rightarrow$ Voir l'article d'É. Mollet. « 


\section{La découverte de la liberté}

soit un universel qui présenterait, à travers le temps, un accomplissement progressif ou des variations quantitatives ou des amputations plus au moins graves, des occultations plus ou moins importantes» (NB, p. 64). Si l'on accepte les termes de ce dilemme, définir rigoureusement la liberté reviendra à lui prêter l'expression d'un universel, sous la forme " la liberté est par nature ceci... ». Reste donc, en portant la même logique à un point extrême, à n'en donner aucune vraie définition.

Cette manière d'envisager le problème intrigue. Pourquoi ne peut-on donner une vraie définition de la liberté, aussi provisoire et circonscrite soit-elle, une définition dans la contingence et la particularité, et non dans la nécessité et l'universalité? En fuyant à trois reprises le besoin de définir un mot (par surcroît, l'un des mots les plus importants de ces cours), Foucault montre le trop d'importance qu'il accorde au geste même de définir, le surprenant prestige qu'il reconnait à la définition et que simultanément il prétend lui nier à tout prix. Ce faisant, avant d'opposer l'universalité à la particularité, il confond celle-ci avec l'ambiguiité, alors qu'aucune raison n'oblige un discours qui se tient sur le plan de celle-là à se contenter de rester dans l'élément de celle-ci, somme toute celui dans lequel reste son discours. Les trois prétendues définitions de la liberté ouvrent la brèche à travers laquelle s'instaure confortablement une ambiguïté embarrassante.

Ce n'est pas tout. À côté des deux fausses alternatives qui accompagnent les deux dernières fausses définitions, il convient de relever une conséquence du traitement que Foucault donne au problème. C'est qu'il devient alors insensé, et impossible, de confronter les degrés de liberté à différentes époques: « jauger la quantité de liberté entre un système et un autre n'a, je crois, de fait pas beaucoup de sens. Et on ne voit pas quel type de démonstration, quel type de jauge ou de mesure on pourrait appliquer. » $(N B$, p. 64.) Foucault pense concrètement à deux régimes: « une monarchie administrative comme celle que connaissait par exemple la France au $\mathrm{XVII}$ e et au XVIII ${ }^{\mathrm{e}}$ siècle » et puis « un régime, disons libéral » (NB, p. 64). Ainsi, entre ce qu'il appelait auparavant l'Âge classique et la Modernité, il serait aussi peu possible d'établir un progrès qu'un déclin de la liberté. Plus fondamentalement, on manquerait de critère pour le faire.

En serait-il vraiment ainsi? Ou bien cet empêchement découle-t-il du refus de donner une définition de la liberté, aussi particulière et contingente soit-elle? Il semble que le serf attaché à la glèbe soit plus libre que l'esclave, et l'ouvrier moderne plus libre que le serf; outre qu'il ne serait 
pas insensé de dire que les citoyens d'un régime démocratique libéral sont plus libres que ceux vivant sous un régime dictatorial. Bien sûr, cela implique de faire appel à un critère et par conséquent à une définition de la liberté. Il n'y a cependant aucune raison pour que l'on voie dans cette définition un universel, ni pour que l'on en fasse l'expression d'une essence. Par ailleurs, rien n'exclut qu'un régime dans lequel les gens sont plus libres (de parler, de circuler, de choisir leurs représentants) soit, par exemple, moins égalitaire qu'un régime dictatorial. Le tout est de ne pas confondre la liberté avec, dans le cas présent, l'égalité. Mais une chose est certaine: l'on n'échappera jamais à cette confusion tant que l'on se refusera de définir les mots que l'on emploie.

\section{La liberté comme production stratégique}

L'importance du double problème de la liberté (problème de savoir ce qu'elle est et quels sont ses liens avec l'exercice du gouvernement) est acquise. Reste à comprendre quel destin Foucault lui réserve. Si l'on revient aux trois « définitions » données, on remarquera non seulement que la liberté, après avoir été découverte en elle-même, est recouverte par les dispositifs de sécurité, qui deviennent l'objet principal de la recherche, mais encore qu'elle est mise sous la dépendance de l'acte de gouverner.

Au premier regard, elle est une condition de possibilité du gouvernement. Foucault le dit à la fin de Sécurité, territoire, population, quand il rappelle combien la liberté est « devenue un élément indispensable à la gouvernementalité elle-même. On ne peut bien gouverner maintenant qu'à la condition qu'effectivement la liberté ou un certain nombre de formes de liberté soient respectées » $(S T P$, p. 361). La même idée réapparaît au début de Naissance de la biopolitique, où on lit que la pratique gouvernementale libérale « ne peut fonctionner que dans la mesure où il y a effectivement un certain nombre de libertés: liberté du marché, liberté du vendeur et de l'acheteur, libre exercice du droit de propriété, liberté de discussion, éventuellement liberté d'expression, etc. » (NB, p.65). Dans cette perspective, la liberté est à la fois partie intégrante des technologies de pouvoir et une composante sans laquelle celui-ci, selon l'art libéral de gouverner, ne peut fonctionner.

Néanmoins, un regard attentif capte un tout autre aspect du problème. La liberté se dévoile alors non pas comme une condition préalable à 


\section{La découverte de la liberté}

l'exercice de ce pouvoir, mais bien plutôt comme un produit de cet exercice, par surcroît un produit stratégiquement élaboré. En premier lieu, elle apparaît comme un produit. Foucault raisonne selon le principe économique de la consommation et de la production: à l'époque du libéralisme, « la nouvelle raison gouvernementale a donc besoin de liberté, le nouvel art gouvernemental consomme de la liberté. Consomme de la liberté, c'est-à-dire qu'il est bien obligé d'en produire. [...] Le libéralisme formule ceci, simplement: je vais te produire de quoi être libre. Je vais faire en sorte que tu sois libre d'être libre» $(N B$, p. 65). Contrairement à ce qu'on aurait pu croire, la liberté n'est pas un a priori du gouvernement, un préalable à lui, qui viendrait donc avant lui. Au contraire, elle accompagne ce gouvernement pour en satisfaire un besoin. Encore accompagner n'est-il pas le verbe qui convient le mieux pour désigner le rapport entre eux, et Foucault insiste sur un autre verbe: produire de la liberté, produire de quoi être libre. En un mot, c'est une affaire de production de l'un par l'autre, celui qui consomme produisant cela même qu'il consomme.

En second lieu, la production de liberté par la pratique gouvernementale libérale se détermine comme stratégique. Le cas qu'illustre le mieux cette idée est la refondation de l'État allemand après la Seconde Guerre mondiale. Le partage de son territoire par les forces de la libération empêche l'Allemagne de se reconstituer politiquement ou juridiquement comme État. À l'embryon d'une autorité précaire, il ne reste alors qu'à instituer et protéger un espace de liberté économique. Dans un cadre institutionnel indéfini surgit non pas un pouvoir de contrainte, typique d'une souveraineté, mais bien la liberté de participer à l'activité économique. «[S]upposons que des individus, en un nombre quelconque, acceptent librement de jouer ce jeu de la liberté économique qui leur est assurée par ce cadre institutionnel. » (NB, p. 84.) Le résultat est qu'ils accepteront, et même réclameront, des mesures qui protègent l'existence et le fonctionnement de cet espace. Le cadre institutionnel en sort renforcé: "Autrement dit, l'institution de la liberté économique va devoir, va pouvoir en tout cas fonctionner, en quelque sorte, comme un siphon, comme une amorce pour la formation de la souveraineté politique. » $(N B$, p. 84.) Foucault entend montrer par là comment la liberté est produite en vue de la densification d'un pouvoir politique raréfié par les circonstances historiques. D'où sa conclusion: dans « cette idée d'une fondation légitimante de l'État sur l'exercice garanti d'une liberté 
économique, $[\ldots]$ on reconnaît très facilement une astuce tactique et stratégique» $(N B$, p. 85).

L'expression abstraite de ce mouvement productif se trouve ici : «En laissant faire les gens, l'institution néolibérale allemande les laisse dire, et elle les laisse faire en grande partie parce qu'elle veut les laisser dire et leur laisser dire quoi? Eh bien, leur laisser dire qu'on a raison de les laisser faire. » (NB, p. 86.) Ce passage éblouissant illustre la vivacité de pensée de Foucault. Il est, par ailleurs, plein d'implications et de résonances. D'une part, il porte en lui le souvenir du dispositif de sexualité, à propos duquel Foucault écrivait que depuis des siècles il nous a enjoint à parler du sexe et à produire sur lui « des discours vrais ${ }^{5} »$. Ici aussi, le discours de la liberté et notre croyance à l'existence première de celleci sont les résultats d'autres stratégies, d'autres dispositifs, mis en place pour produire et cette liberté et ce discours et cette croyance. D'autre part, ce passage fait écho à la formule « laissez faire, laissez passer », étudiée au début de Sécurité, territoire, population (p. 43) et reprise dans Naissance de la biopolitique (p. 22). En toute rigueur, il clôt cette étude, en prétendant que notre «dire » des libertés qu'on nous laisse, est un « dire » qu'on nous laisse également, de plus un « dire » auquel on nous invite, qu'on nous fait dire. C'est cela le sens du vouloir laisser dire que Foucault met au centre de son analyse. Si l'on dit qu'on est libre de faire, c'est en bonne partie parce que ceux qui nous laissent faire veulent aussi qu'on le dise. Plus généralement encore, si l'on dit qu'on est libre, c'est que quelqu'un veut nous le laisser dire, c'est qu'on veut qu'on le dise. Astuce stratégique, déclare Foucault, qui a pour but immédiat de renforcer l'institution elle-même, sa légitimité et son action, comme le consensus qui les soutiendra.

Prise dans ces mailles, la liberté ambiguë nous laisse dans l'impasse. D'abord, n'étant rien d'autre qu'un corrélatif des dispositifs de sécurité, elle nous apparaît comme dépendante de la mise en place de ces dispositifs, dont elle est « une des faces, un des aspects, une des dimensions » (STP, p. 50). Ensuite, étant non pas acceptée par le gouvernement libéral et encore moins imposée à lui ou conquise par les luttes théoriques et pratiques, mais au contraire produite par ce gouvernement comme un besoin qu'il doit satisfaire, la liberté reste sous la dépendance semble-t-

5. VS, coll. « Tel», 1998 [1976], p. 91 


\section{La découverte de la liberté}

il exclusive de l'acte de gouverner. Tout nous apparaît ainsi comme le résultat compréhensible d'un art qui se développe de façon naturelle, à mesure qu'il a à résoudre les problèmes de gouvernement d'une ville ou d'un État. Enfin, quand on apprend que cette production est une astuce tactique et stratégique d'une pratique de gouvernement qui cherche avant tout par là à se légitimer, on se demande si la liberté ne serait en fait rien d'autre que cela, et on s'interroge sur les pentes théoriques au long desquelles l'analyse a pu glisser jusqu'à se faire de la liberté une conception si étroite et si pauvre.

\section{La biopolitique, promesse non tenue}

Arrivé là, tout lecteur de Foucault s'écriera que derrière ce processus apparemment tranquille, il faut savoir, toujours et encore, « entendre le grondement de la bataille ${ }^{6} \gg$. Mais à quelles conditions ce grondement est-il audible? Sur quel terrain cette bataille se déroule-t-elle? Certes non pas sur celui qui sépare la sécurité de la liberté, comme on vient de le voir, mais sur celui qu'ouvre devant nous le retour au thème de la résistance. La leçon du $1^{\text {er }}$ mars 1978 devient alors incontournable, dans la place singulière qu'elle occupe dans l'ensemble des deux cours.

Foucault y fait l'histoire des mouvements d'opposition au pouvoir pastoral, qu'il traite comme des mouvements de contre-conduite $\rightarrow$ voir l'article d'E. Taïeb $\nleftarrow$. Sa thèse est la suivante: «S'il est vrai que le pastorat est un type de pouvoir bien spécifique qui se donne pour objet la conduite des hommes [...], je crois que, corrélativement à cela, sont apparus des mouvements tout aussi spécifiques que ce pouvoir pastoral [...] qui sont des résistances, des insoumissions, quelque chose qu'on pourrait appeler des révoltes spécifiques de conduite [...].» (STP, p. 198.) Voilà, sans équivoque, le retour au thème de la résistance. Et on pourrait même prétendre que ce passage est une application directe, au terrain concret du pastorat chrétien, du principe énoncé dans $L a$ volonté de savoir, selon lequel «là où il y a pouvoir, il y a résistance ${ }^{7}$ ». Nous sommes bien sur le point de redécouvrir la dimension de la lutte.

\footnotetext{
6. $S P$, p. 315 .

7. VS, coll. « Tel », p. 125, passage que cite aussi Michel Senellart, STP, p. 221, n. 5.
} 
Seulement, ce n'est pas à un simple retour qu'on a affaire, mais à un déplacement décisif ${ }^{8}$. Une voie inouie s'ouvre dans la pensée de Foucault, selon laquelle la résistance n'est plus suscitée par le pouvoir, mais par le gouvernement, de telle sorte qu'elle prend elle-même à son tour la forme d'un autre gouvernement, d'une contre-conduite qui est un gouvernement de soi. Le glissement du pouvoir au gouvernement n'est pas anodin et représente une nouveauté majeure de ces cours, les mots gouvernement et gouvernementalité devançant ceux de pouvoir et de pouvoir-savoir. En outre, ce glissement prépare le saut conceptuel vers ce qui sera, quelques années plus tard, le gouvernement de soi et des autres, le croisement des régions éthique et politique de l'expérience.

Le surgissement des contre-conduites dans le discours de Foucault mériterait une analyse circonstanciée que nous ne pouvons faire ici. En elles s'esquisse déjà l'idée (exprimée plus tard) que «l'exercice du pouvoir consiste à "conduire des conduites" » et qu'il est donc «une action sur des actions ${ }^{9}$ »; à leur sujet, Foucault trouve, à notre grand étonnement, un critère pour jauger la quantité d'intervention d'un pouvoir concret sur l'existence spirituelle des individus à deux époques distinctes (avant et après la Réforme et la Contre-Réforme ${ }^{10}$ ), contrairement à ce qu'il avait fait avec la liberté, comme on l'a vu; enfin, autrement plus important, les contre-conduites ouvrent la voie à une autre sorte de liberté, plus éthique que politique: la découverte de la liberté de choix éthique sera la réponse à l'échec de la liberté dans la politique. Il y a, dans cette transition d'un domaine à l'autre, une solidarité et une logique.

$\mathrm{Si}$ bien que cette leçon isolée explique en partie l'abandon de la biopolitique. En étudiant le libéralisme, Foucault a découvert les libertés: elles sont des possibilités de choisir, qu'il considère comme étant produites par l'art de gouverner libéral. Au même moment, il a découvert les contre-conduites: elles sont des possibilités de choisir qui s'insurgent contre une certaine forme d'être conduit ou gouverné. Après ces découvertes, continuer l'étude de la biopolitique serait persévérer dans la description du recouvrement des libertés politiques par les dispositifs de sécurité et renoncer à explorer l'autre piste qui se dessinait, celle de

\footnotetext{
8. Je reprends à mon compte des hypothèses avancées dans la journée d'études de Labyrinthe par Étienne Balibar, Bruno Karsenti et Hélène L'Heuillet.

9. Foucault, « Le sujet et le pouvoir », op. cit., p. 237. Voir STP, p. 198, 203 et 205.

10. Voir STP, p. 235.
} 


\section{La découverte de la liberté}

la liberté du choix éthique. C'est encore le chemin de la biopolitique que poursuit Foucault jusqu'à la fin de ces deux cours. Puis, il y renoncera.

Et c'est bien d'un abandon qu'il s'agit, l'étude de la biopolitique étant, au dire de Foucault, une promesse non tenue. Lorsque dans le cours de 1978-1979 il synthétise son projet de recherche pour l'année, il termine en déclarant: "Et alors, enfin, si la chance me sourit, on arrivera au problème de la biopolitique et au problème de la vie.» $(N B$, p. 80.) Certes, on trouve tout au long des deux cours des références à cette notion, mais jamais elle n'est considérée en elle-même. Foucault reconnaîtra peu après que «le cours de cette année a finalement été consacré, en son entier, à ce qui devait n'en former que l'introduction» ( $N B$, « Résumé du cours », p. 323). Par ailleurs, le cours suivant, de 1979-1980, intitulé « Du gouvernement des vivants », traitera d'un tout autre sujet, des techniques d'aveu. L'intérêt porté à la biopolitique se dissipe sans jamais donner lieu à une recherche aboutie. Quand Foucault veut raconter la naissance de la biopolitique, il n'en fait qu'une introduction. Et lorsque l'introduction est finie et qu'il peut enfin entrer en matière, il change de sujet.

On comprend la fin étrange d'un mot qui pourtant semblait taillé pour devenir un objet théorique crucial. Si l'analyse de la biopolitique est à deux reprises impossible et en définitive abandonnée, c'est qu'elle soulève une difficulté à laquelle elle ne peut apporter de solution, qu'elle est impuissante à répondre au problème qui se pose en elle: celui de la liberté. Tel qu'il a été exposé dans Surveiller et Punir et dans La volonté de savoir, tel qu'il continue d'être traité dans les deux cours nonobstant certaines métamorphoses, il ne peut être résolu ni dans le domaine de la biopolitique ni dans celui, plus vaste, du pouvoir. Foucault pensera à un moment qu'il n'y a de la place pour la liberté que dans le monde éthique, raison pour laquelle il se passionnera pour les Anciens et pour ce qu'ils ont pu dire et faire des plaisirs sexuels. Et c'est là qu'on touche à la vraie raison de son retour à l'Antiquité: de la biopolitique, Foucault s'acheminera vers la bioéthique (vers la technê tou biou), deux cadres pour penser la vie, mais surtout deux espaces pour chercher la liberté. 